\title{
Retinal thickness as a marker of disease progression in longitudinal observation of patients with Wolfram syndrome
}

\author{
Agnieszka Zmyslowska ${ }^{1}$ Wojciech Fendler ${ }^{2,3}$ - Arleta Waszczykowska ${ }^{4}$. \\ Anna Niwald ${ }^{5} \cdot$ Maciej Borowiec $^{6} \cdot$ Piotr Jurowski $^{4} \cdot$ Wojciech Mlynarski $^{1}$
}

Received: 10 July 2017 / Accepted: 16 August 2017 / Published online: 30 August 2017

(c) The Author(s) 2017. This article is an open access publication

\begin{abstract}
Aims Wolfram syndrome (WFS) is a recessively inherited monogenic form of diabetes coexisting with optic atrophy and neurodegenerative disorders with no currently recognized markers of disease progression. The aim of the study was to evaluate retinal parameters by using optical coherence tomography (OCT) in WFS patients after 2 years of followup and analysis of the parameters in relation to visual acuity. Methods OCT parameters and visual acuity were measured in 12 WFS patients and 31 individuals with type 1 diabetes. Results Total thickness of the retinal nerve fiber layer (RNFL), average retinal thickness and total retinal volume decreased in comparison with previous OCT examination. Significant decreases were noted for RNFL (average difference $-17.92 \mu \mathrm{m} \mathrm{95 \%} \mathrm{CI}-30.74$ to $-0.10 ; p=0.0157$ ), macular average thickness (average difference $-5.38 \mu \mathrm{m} 95 \%$ CI -10.63 to $-2.36 ; p=0.0067$ ) and total retinal volume
\end{abstract}

Managed By Antonio Secchi.

Wojciech Mlynarski

wojciech.mlynarski@umed.lodz.pl

1 Department of Pediatrics, Oncology, Hematology and Diabetology, Medical University of Lodz, Sporna Str. 36/50, 91-738 Lodz, Poland

2 Department of Biostatistics and Translational Medicine, Medical University of Lodz, Lodz, Poland

3 Department of Radiation Oncology, Dana-Farber Cancer Institute, Boston, MA, USA

4 Department of Ophthalmology and Vision Rehabilitation, Medical University of Lodz, Lodz, Poland

5 Outpatient Clinic of Ophthalmology, Central Clinical Hospital, Lodz, Poland

6 Department of Clinical Genetics, Medical University of Lodz, Lodz, Poland (average difference $-0.15 \mathrm{~mm}^{3} 95 \% \mathrm{CI}-0.30$ to -0.07 ; $p=0.0070$ ). Central thickness remained unchanged (average difference $1.5 \mu \mathrm{m} \mathrm{95 \%} \mathrm{CI}-7.61$ to $10.61 ; p=0.71$ ). Visual acuity of WFS patients showed a strong negative correlation with diabetes duration $(R=-0.82 ; p=0.0010)$. After division of WFS patients into two groups (with low-vision and blind patients), all OCT parameters except for the RNFL value were lower in blind WFS patients.

Conclusions OCT measures structural parameters and can precede visual acuity loss. The OCT study in WFS patients should be performed longitudinally, and serial retinal examinations may be helpful as a potential end point for future clinical trials.

Keywords Monogenic diabetes - Wolfram syndrome . Ophthalmology complications $\cdot$ Retina

\section{Introduction}

Wolfram syndrome (WFS) (OMIM 222300) is caused by recessive mutations mainly in the WFS1 gene on chromosome $4 \mathrm{p} 16.1[1,2]$. Wolframin, the protein product of WFS1, is an important part of the endoplasmic reticulum (ER) membrane and its loss of function in neuroendocrine cells increases ER stress, leading to apoptosis and neurodegeneration [3]. At present there is no causal treatment for WFS, and the disease leads to premature death of the patients [4].

The first clinical signs of WFS are diabetes mellitus (DM) and optic atrophy (OA). Typically, the onset of insulindependent DM precedes the diagnosis of OA, by a margin of 4 years on average [5-7]. Other features of the syndrome, which develop as the patient gets older, are: progressive blindness and deafness, diabetes insipidus, urodynamic and endocrinological disorders, and psychiatric and neurological 
abnormalities [8]. In addition to OA, other vision abnormalities (maculopathy, retinopathy and cataract) may emerge, contributing to impairment and loss of vision $[9,10]$. Moreover, very recent studies on Wfs1-deficient mice disclosed significant dysfunction of the visual system, associated with ER stress in the retina [11]. These facts support the need to perform studies focused on neurodegenerative changes in the retina among patients with WFS. The best way to evaluate retinal integrity is by using optical coherence tomography (OCT), which can non-invasively detect pathology of the optic nerves and also images the posterior pole of the eye [12]. Therefore, we focused on the clinical features of WFS and combined them with the OCT results suggesting that a retinal thinning could serve as a biomarker of WFS disease progression [13]. Over time, visual acuity of WFS patients becomes worse which can exclude accurate estimation of some OCT parameters [14]. However, a long-term retina evaluation seems to be clinically useful. Thus, the aim of the study was to evaluate retinal parameters by using OCT after 2 years of follow-up and analysis of the parameters in relation to visual acuity in patients with WFS.

\section{Materials and methods}

Before initiating the study, the Bioethics Committee of the Medical University of Lodz approved the study protocol and all patients or parents of underage participants expressed written consent for participating in the study. The present study included two groups: 12 patients with WFS and a control group composed of 31 individuals with type 1 diabetes (T1D). Among the 12 patients with WFS, 10 were a prospectively followed-up group whose initial data were published previously [13]. The remaining two individuals at follow-up analysis were newly recruited WFS patients without prior OCT examinations. The average duration of follow-up in the WFS group was $22 \pm 2.2$ months. The T1D group was recruited as controls for the WFS individuals among children treated in the study center from children consecutively hospitalized within the Department of Pediatrics, Oncology, Hematology and Diabetology between October 2015 and January
2016. Only children with duration of diabetes longer than 3 years admitted for routine metabolic control assessment were recruited to the study.

Diabetes was recognized typically, according to WHO criteria. At onset of T1D, autoantibodies and decreased C-peptide level were detected in all patients. HbA1c was determined by high-performance liquid chromatography (HPLC) using the Bio-Rad VARIANT ${ }^{\text {TM }}$ Hemoglobin A1c Program (Bio-Rad Laboratories, Inc. Hercules, CA, USA) with its values represented as percentages and $\mathrm{mmol} / \mathrm{mol}$.

All WFS patients had DM recognized according to WHO criteria, OA recognized using visual evoked potentials (VEP) and/or MRI, and confirmed during the first OCT study. Detailed characteristics of the study groups are shown in Table 1. The causative mutations in the WFS1 gene detected in all WFS patients were recognized as described previously [14].

\section{OCT study}

All first OCT examinations in WFS patients were conducted in the Clinic of Ophthalmology of the Central University Hospital in Lodz, Poland, with the Topcon 3D Optical Coherence Tomograph 2000 (Fastmap version 8.11.003.04, Japan). The second OCT study was performed in the patients from the study and control groups in the Department of Ophthalmology and Vision Rehabilitation of the Medical University of Lodz, Poland, with Topcon 3D Optical Coherence Tomograph 1000 (MARK II, version 3.51, Topcon Inc., Paramus, NJ, USA). All studies were carried out with subjects in a resting position after an average of 15-20 min after inducing mydriasis and evaluated independently by two experienced ophthalmologists. We determined the following parameters: total thickness of the retinal nerve fiber layer (RNFL), average retinal thickness, central thickness and total retinal volume using 3D disk and 3D macula scans with a $6 \times 6 \mathrm{~mm}$ area. Owing to absence of visual fixation in patients with $\mathrm{OA}$, three parameters were measured in all WFS patients: average retinal thickness, central thickness and total volume. Averages from measurements in both eyes were calculated and used for further analysis.

Table 1 General characteristics of the studied groups

\begin{tabular}{|c|c|c|c|c|c|}
\hline Group & Sex M/F & $\begin{array}{l}\text { Age at OCT study and visual acuity } \\
\text { examinations average } \pm \text { SD (years) }\end{array}$ & $\begin{array}{l}\text { Age at diabetes diagnosis } \\
\text { average } \pm \mathrm{SD} \text { (years) }\end{array}$ & $\begin{array}{l}\text { Duration of diabetes } \\
\text { average } \pm S D \text { (years) }\end{array}$ & $\begin{array}{l}\text { HbA1c average } \pm \text { SD } \\
(\% / \mathrm{NGSP} / \mathrm{mmol} / \mathrm{mol} \\
\text { IFCC })\end{array}$ \\
\hline $\begin{array}{l}\text { WFS } \\
N=12\end{array}$ & $3 / 9$ & $18.58 \pm 4.48$ & $6.19 \pm 2.99$ & $12.38 \pm 4.86$ & $7.86 \pm 1.12 / 62 \pm 12.5$ \\
\hline $\begin{array}{l}\text { T1D } \\
N=31\end{array}$ & $13 / 18$ & $12.63 \pm 3.36$ & $6.70 \pm 3.58$ & $5.82 \pm 2.88$ & $7.29 \pm 0.62 / 56 \pm 7$ \\
\hline
\end{tabular}

WFS Wolfram syndrome, $T 1 D$ type 1 diabetes, $S D$ standard deviation 


\section{Visual acuity}

All the patients underwent at follow-up an assessment of best spectacle-corrected visual acuity (BSCVA) for distant vision measured using Snellen charts at a decimal scale (LCD Frey CP-400, Frey Sp.J., Piaseczno, Poland). The examination was conducted independently by two experienced ophthalmologists. All T1D patients fulfilled the criteria for correct vision $(>0.3)$. Depending on the results of visual acuity, WFS patients were divided into patients with low-vision $(0.05-0.3)$ and blind patients $(<0.05)$.

\section{Statistical analysis}

Paired comparisons were performed with a Wilcoxon's signed rank test for matched samples. Correlations were evaluated with a Spearman's rank correlation test. Generalized linear regression model was used to adjust for confounding factors according to the standard methodology of accounting for the impact of such variables as described in [15]. STATISTICA 13.0 software (Statsoft, Tulsa, OK,
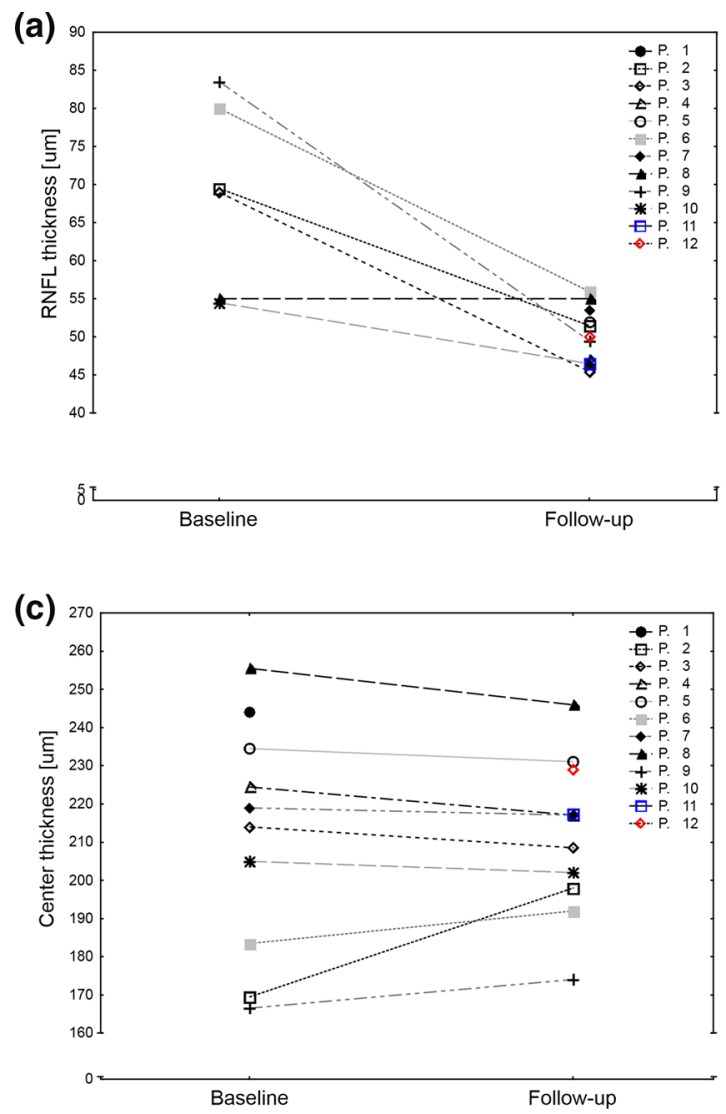

Fig. 1 OCT parameters observed in patients with Wolfram syndrome at the first examination and 2-year follow-up observation. Significant decreases were noted for RNFL (average difference $-17.92 \mu \mathrm{m}$ $95 \% \mathrm{CI}-30.74$ to $-0.10 ; p=0.0157$ (a), macular average thickness (average difference $-5.38 \mu \mathrm{m} \mathrm{95 \%} \mathrm{CI}-10.63$ to $-2.36 ; p=0.0067$
USA) was used for analysis. A type 1 error probability of 0.05 was chosen as the threshold of statistical significance.

\section{Results}

Analysis of OCT data showed that in patients with WFS, significant thinning of the RNFL, average retinal thickness and total retinal volume decreased in comparison with the previous examination (Fig. 1a-c). Central thickness remained unchanged (Fig. 1d). Average decrease of RNLF equaled $12.90 \% / y e a r$ 95\% CI $4.22-21.52 \%$, of average macular thickness $1.54 \%$ year $95 \%$ CI $0.535-2.55 \%$ and $1.53 \%$ year $95 \%$ CI $0.51-2.55 \%$ of total retinal volume. All four studied OCT parameters of patients with WFS were consistently lower than ones observed in patients with T1D (Table 2) and they-except for the RNFL value - correlated with OCT parameters from the first examination 2 years ago (Table 3). Then, we evaluated visual acuity of patients with WFS at follow-up examination. Median value equaled 0.083 with three individuals showing complete lack of vision apart from

(b)
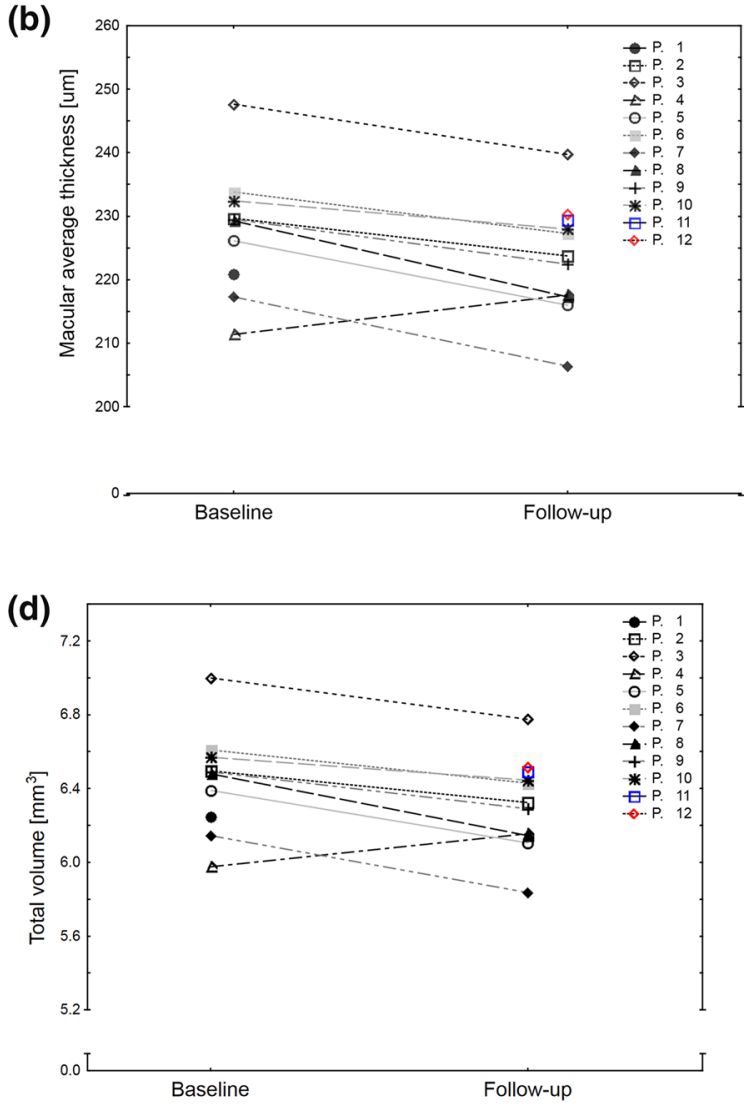

(b) and total retinal volume (average difference $-0.15 \mathrm{~mm}^{3} 95 \%$ CI -0.30 to $-0.07 ; p=0.0070$ (c). Central thickness remained unchanged (average difference $1.5 \mu \mathrm{m} \mathrm{95 \%} \mathrm{CI}-7.61$ to 10.61 ; $p=0.71(\mathbf{d})$ 
Table 2 Comparison of OCT data between patients with WFS and T1D at follow-up study

\begin{tabular}{|c|c|c|c|c|c|}
\hline & \multicolumn{2}{|l|}{ WFS } & \multicolumn{2}{|l|}{ T1D } & \multirow[t]{2}{*}{$p$ level* } \\
\hline & Average & SD & Average & SD & \\
\hline RNFL & 50.27 & 3.64 & 94.59 & 13.74 & 0.000001 \\
\hline Macular average thickness $(\mu \mathrm{m})$ & 223.48 & 8.96 & 285.31 & 13.72 & 0.000001 \\
\hline Center thickness $(\mu \mathrm{m})$ & 212.00 & 20.10 & 259.00 & 20.00 & 0.000005 \\
\hline Total macular volume $\left(\mathrm{mm}^{3}\right)$ & 6.32 & 0.25 & 10.27 & 0.49 & 0.000001 \\
\hline
\end{tabular}

WFS Wolfram syndrome, T1D type 1 diabetes, $S D$ standard deviation, $R N F L$ retinal nerve fiber layer

* Adjusted for age and duration of diabetes
Table 3 Correlations between OCT parameters at the first examination and follow-up study in the patients with Wolfram syndrome

\begin{tabular}{llr}
\hline & $R$ & \multicolumn{1}{c}{$p$ level } \\
\hline RNFL & 0.3143 & 0.5441 \\
Macular average thickness $(\mu \mathrm{m})$ & 0.8833 & 0.0016 \\
Center thickness $(\mu \mathrm{m})$ & 0.9791 & $<0.0001$ \\
Total macular volume $\left(\mathrm{mm}^{3}\right)$ & 0.8833 & 0.0016 \\
\hline
\end{tabular}

$R N F L$ retinal nerve fiber layer

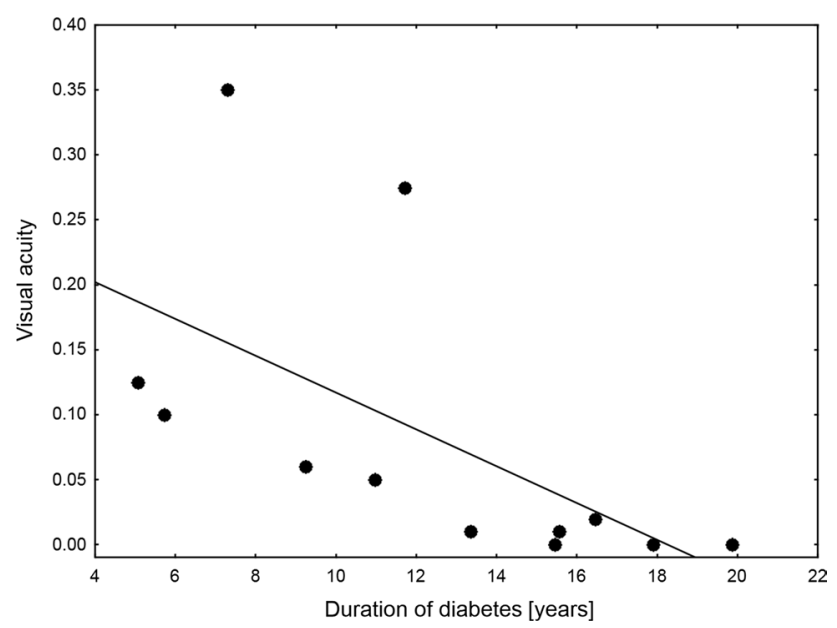

Fig. 2 Correlations between visual acuity measured at follow-up and duration of diabetes in Wolfram syndrome patients a sense of light in both eyes. Visual acuity of patients with WFS showed a strong negative correlation with the duration of diabetes (Spearman's $R=-0.82 ; p=0.0010$, Fig. 2) and correlated with patients' age, but the difference did not reach statistical significance $(R=-0.44 ; p=0.1558)$. Finally, we divided WFS patients to the patients with low-vision and blind patients. Among OCT parameters, only RNFL remained similar in the subgroups, whereas other retinal parameters differed significantly (Table 4).

\section{Discussion}

For the first time, our study shows that average retinal thickness and total retinal volume of OCT parameters in WFS patients decrease slower than RNFL value. Moreover, since the retinal parameters in WFS patients differed significantly from those observed in patients with T1D after adjustment for the age and duration of diabetes, it is very likely that the changes noted in OCT examinations of WFS patients are the direct consequence of WFS progression rather than of diabetes. It seems, however, that there is a limit value of OCT parameters in WFS patients below of which no retinal thinning progression is observed. Thus, the results show the greatest usefulness of OCT measurements on the early stage of WFS as potential biomarkers of WFS progression. Similar observations were performed by Hoekel et al. evaluating the optical phenotype at a relatively early stage of WFS and making an attempt of correlation with the severity of the
Table 4 Comparison of OCT data between Wolfram syndrome patients with lowvision and blind patients

\begin{tabular}{lcclll}
\hline Parameter & $\begin{array}{l}\text { Average } \\
\text { Patients } \\
\text { with low } \\
\text { vision }\end{array}$ & $\begin{array}{l}\text { SD } \\
\text { Patients } \\
\text { with low } \\
\text { vision }\end{array}$ & $\begin{array}{l}\text { Average } \\
\text { Blind patients }\end{array}$ & $\begin{array}{l}\text { SD } \\
\text { Blind patients }\end{array}$ & $p$ level \\
\hline RNFL & 49.25 & 3.99687 & 51.5 & 3.1225 & 0.333284 \\
Macular average thickness $(\mu \mathrm{m})$ & 228.4417 & 6.13526 & 217.52 & 8.50512 & 0.035218 \\
Center thickness $(\mu \mathrm{m})$ & 198.6667 & 14.93876 & 228 & 12 & 0.006382 \\
Total macular volume $\left(\mathrm{mm}^{3}\right)$ & 6.4592 & 0.17226 & 6.151 & 0.24214 & 0.035724 \\
\hline
\end{tabular}

$R N F L$ retinal nerve fiber layer, $S D$ standard deviation 
disease. Decrease in RNFL during OCT examination correlated in the study with the severity of physical symptoms. However, a lack of correlation between age and reduction in visual acuity was noted making the RNFL value reliable but only the initial marker of WFS progression [16]. Interestingly, our patients at follow-up analysis were an average of 4 years older than patients presented by Hoekel et al. Importantly, in four WFS patients described by Grenier et al. [17] who were about 4.5 years older than our patients, the RNFL thickness was only slightly lower in comparison with our patients.

This is worth noting that a visual fixation by the patient is necessary to measure RNFL during OCT. Thus, the approach cannot be used in some elderly, partially sighted or blind patients. In the present study, by the division of WFS patients depending on the vision acuity we confirmed that RNFL did not differ between groups with low vision and blind, whereas the average retinal thickness and retinal total volume were significantly lower and center thickness was greater in the blind patients. Thus, the best option for the advanced stage of the WFS is to evaluate more stable retinal OCT parameters which could be measured independently of sight fixation.

During follow-up of OCT examination in WFS patients, the significant declines were noted in RNFL, total retinal volume and average thickness, whereas central retinal thickness did not change within 2 years of observation. We can speculate that this finding may be related to the increased risk of maculopathy in these patients or to cellular edema resulting from increased cellular volume due to ER stress.

This also suggested that a loss of retinal neurons is initially reflected by the peripheral thinning of the retinal lining of the eye, while the central thickness remained, at least initially, unaffected. These results are in line with available data about the reduced thickness of the RNFL assessed in OCT studies of neurodegenerative changes occurring in the brain of patients with multiple sclerosis and sleep apnea [18, 19]. Macular thickness is already used as a marker in monitoring treatment of patients with Parkinson's disease [20], Alzheimer's disease [21] and age-related macular degeneration (AMD) [22].

Our study also has some limitations. Most importantly, we were not able to recruit a suitably large group of patients with WFS. This is because there are very few such individuals in the Polish cohort recruited through the EURO-WABB rare diseases project $[14,23]$. In addition, the first and second OCT studies were conducted on two different devices. However, both were still performed on Topcon 3D Optical Coherence Tomograph, while literature data indicate an objective signal quality of retinal OCT images regardless of the device [24]. Moreover, due to a lack of visual fixation in blind and with very low-vision WFS patients, RNFL thickness was evaluated only in some patients.
Looking at our results, OCT measures structural parameters and can precede visual acuity loss. It should be emphasized that the OCT study in WFS patients should be performed longitudinally, and serial retinal examinations may be helpful as a potential end point for future clinical trials.

Acknowledgements This study is supported by the National Science Centre Grants Nos. 2014/15/B/NZ5/01579 and 2015/19/B/NZ5/02243 and the Foundation for Polish Science IMPULS Grant No. 38/UD/ SKILLS/2015 and funds of the Medical University of Lodz No.: 503/1090-01/503-11-003 and 502-03/1-090-01/502-14-175.

\section{Compliance with ethical standards}

Conflict of interest The authors declare that they have no conflict of interest.

Ethical standards All procedures followed were in accordance with the ethical standards of the responsible committee on human experimentation (institutional and national) and with the Helsinki Declaration of 1975 , as revised in 2008 .

Informed consent Informed consent was obtained from all patients for being included in the study.

Open Access This article is distributed under the terms of the Creative Commons Attribution 4.0 International License (http://creativecommons.org/licenses/by/4.0/), which permits unrestricted use, distribution, and reproduction in any medium, provided you give appropriate credit to the original author(s) and the source, provide a link to the Creative Commons license, and indicate if changes were made.

\section{References}

1. Ghahraman M, Abbaszadegan MR, Vakili R et al (2016) Identification of four novel mutations of the WFS1 gene in Iranian Wolfram syndrome pedigrees. Acta Diabetol 53:899-904

2. Rondinelli M, Novara F, Calcaterra V, Zuffardi O, Genovese S (2015) Wolfram syndrome 2: a novel CISD2 mutation identified in Italian siblings. Acta Diabetol 52:175-178

3. Fonseca SG, Ishigaki S, Oslowski CM et al (2010) Wolfram syndrome 1 gene negatively regulates ER stress signaling in rodent and human cells. J Clin Invest 120:744-755

4. Kinsley BT, Swift M, Dumont RH, Swift RG (1995) Morbidity and mortality in the Wolfram syndrome. Diabetes Care 18:1566-1570

5. Zmyslowska A, Borowiec M, Fichna P et al (2014) Delayed recognition of Wolfram syndrome frequently misdiagnosed as type 1 diabetes with early chronic complications. Exp Clin Endocrinol Diabetes 122:35-38

6. Zmyslowska A, Fendler W, Szadkowska A et al (2015) Glycemic variability in patients with Wolfram syndrome is lower than in type 1 diabetes. Acta Diabetol 52:1057-1062

7. Pedroso JL, Lucato LT, Kok F, Sallum J, Barsottini OG, Oliveira AS (2015) Association of optic atrophy and type 1 diabetes: clinical hallmarks for the diagnosis of Wolfram syndrome. Arq Neuropsiquiatr 73:466-468

8. Barrett TG, Bundey SE, Macleod AF (1995) Neurodegeneration and diabetes: UK nationwide study of Wolfram (DIDMOAD) syndrome. Lancet 346:1458-1463 
9. Al-Till M, Jarrah NS, Ajlouni KM (2002) Ophthalmologic findings in fifteen patients with Wolfram syndrome. Eur J Ophthalmol 12:84-88

10. Dhalla MS, Desai UR, Zuckerbrod DS (2006) Pigmentary maculopathy in a patient with Wolfram syndrome. Can J Ophthalmol 41:38-40

11. Bonnet Wersinger D, Benkafadar N, Jagodzinska J et al (2014) Impairment of visual function and retinal ER stress activation in Wfs1-deficient mice. PLoS ONE 9:e97222

12. Al-Mujaini A, Wali UK, Azeem S (2013) Optical coherence tomography: clinical applications in medical practice. Oman Med J 28:86-91

13. Zmyslowska A, Fendler W, Niwald A et al (2015) Retinal thinning as a marker of disease progression in patients with Wolfram syndrome. Diabetes Care 38:e36-e37

14. Zmyslowska A, Borowiec M, Antosik K et al (2011) Wolfram syndrome in the Polish population: novel mutations and genotypephenotype correlation. Clin Endocrinol (Oxf) 75:636-641

15. Rosner B (2011) Fundamentals of biostatistics, Chapter 12.10, 7th edn. Thomson-Brooks/Cole, Belmont. ISBN-10: 0538733497

16. Hoekel J, Chisholm SA, Al-Lozi A, Hershey T, Tychsen L, Washington University Wolfram Study Group (2014) Ophtalmologic correlates of disease severity in children and adolescents with Wolfram syndrome. J AAPOS 18:461-465

17. Grenier J, Meunier I, Daien V et al (2016) WFS1 in optic neuropathies: mutation findings in nonsyndromic optic atrophy and assessment of clinical severity. Ophthalmology 123:1989-1998
18. Fjeldstad AS, Carlson NG, Rose JW (2012) Optical coherence tomography as a biomarker in multiple sclerosis. Expert Opin Med Diagn 6:593-604

19. Huseyinoglu N, Ekinci M, Ozben S, Buyukuysal C, Kale MY, Sanivar HS (2014) Optic disc and retinal nerve fiber layer parameters as indicators of neurodegenerative brain changes in patients with obstructive sleep apnea syndrome. Sleep Breath 18:95-102

20. Satue M, Seral M, Otin S et al (2014) Retinal thinning and correlation with functional disability in patients with Parkinson's disease. Br J Ophthalmol 98:350-355

21. Iseri PK, Altinas O, Tokay T, Yuksel N (2006) Relationship between cognitive impairment and retinal morphological and visual functional abnormalities in Alzheimer disease. J Neuroophthalmol 26:18-24

22. Michalewski J, Nawrocki J, Izdebski B, Michalewska Z (2014) Morphological changes in spectral domain optical coherence tomography guided bevacizumab injections in wet age-related macular degeneration, 12-months results. Indian J Ophthalmol 62:554-560

23. Farmer A, Ayme S, de Heredia ML et al (2013) EURO-WABB: an EU rare diseases registry for Wolfram syndrome, Alstrom syndrome and Bardet-Biedl syndrome. BMC Pediatr 13:130

24. Huang Y, Gangaputra S, Lee KE et al (2012) Signal quality assessment of retinal optical coherence tomography images. Invest Ophthalmol Vis Sci 53:2133-2141 